\section{Temporal and spatial characteristics of selective encoding from visual displays*}

\author{
CHARLES W. ERIKSEN and JAMES E. HOFFMAN \\ University of Illinois, Champaign/Urbana, Illinois 61820
}

The time required for Ss to voice a target letter in a visual display was studied as a function of the spatial proximity of two kinds of noise elements (letters or disks) to the target and as a function of when the noise elements were presented following the onset of the target letter. The results were not consistent with a focusing model of attention or selective encoding. Instead, there appears to be a small area in the visual field (about $1 \mathrm{deg}$ of angle) in which all stimuli are processed in detail.

In prior work, we have been investigating the selective processes in visual perception by which one stimulus is encoded and/or reported from among a number of equally potent stimuli. The basic experimental technique presents simultaneously a visual display containing a circular arrangement of letters with a line indicator designating the target letter that $S$ is to report.

We have found (Eriksen \& Hoffman, 1972 ) that the time required for the $S$ to voice the target letter progressively decreases as the indicator line designating the position of the target letter precedes the display by longer and longer intervals, up to at least 150 msec. Also, the Ss' voicing latency (reaction time) increases as the number of letters in the display increases from 4 to 12 , and there is some indication that the RT difference between 4- and 8-letter and 4- and 12-letter displays decreases as the indicator precedes the display out to at least 150 msec.

There are various ways in which a leading position indicator could act to reduce reaction time. Obviously the indicator itself must be perceived, or, in terms of the current vernacular, the selective encoding must be programmed. The perception time for the indicator can be presumed to have some average value, with a distribution reflecting its variability from trial to trial. If the time required to perceive an indicator is comparable to that required to perceive a letter, the distribution of these latencies can encompass a duration in excess of 100 msec (Erikeen \& Eriksen, 1971, 1972). The average reaction time to the target letter would be expected to show a decrease until the range of perception time latencies for the indicator had been exceeded.

The number of noise elements in

*This inventigation was supported by United 8 tates Public Health Service Research Grant MH-1206 and United States Research Grant MH-1206 and United States
Public Health Service Research Career Program Award No. K6-MH-22014. the display could influence reaction time in either of two nonexclusive ways. The greater the number of noise elements in a display, the greater the information conveyed by the line indicator. If it takes longer to perceive an indicator designating 1 of 12 possible positions than it does to perceive one designating 1 of 4 , we would have reduced the affect of the number of noise elements to the familiar problem of stimulus uncertainty on reaction time. This possibility, however, seems somewhat unlikely in view of the lack of success in demonstrating that the positional uncertainty in the stimulus systematically influences performance (Barber \& Folkard, 1972).

A second possibility is that the noise elements in the display interfere in some manner with the perception of the line indicator. The greater the number of noise elements, the greater the interference. The nature of this interference is unspecified but might be a low-level parallel processing of the noise elements along with the indicator. Thus, perception of the indicator is slowed by draining of available energy into thisparallel task. This would be similar to the limited-capacity parallel processing model envisaged by Rummelhart (1970).

The above possibilities concerning the perception time for the indicator may be sufficient to account for the reduction in reaction time to the target letter, but it is also possible that other processes may be involved. If stimulus selection in visual perception worked like a well designed computer, then once the selection information had been programmed, the speed or accuracy with which the designated stimulus was read out would be of adjacent stimuli. But stimulus selection in visual perception may not be this efficient. Selective encoding may involve a bracketing or focusing-in process that in itself requires time. The time required to independent of the number or density focus upon a specific location would be proportional to the precision of that location in the visual field. Such a focusing process likely would be sensitive to not only the number of noise elements in the display but also to their physical spacing or proximity. The more elements in the display and the closer their proximity, the more precise the focusing would have to be to encode only the designated item. Prior results (Eriksen \& Hoffman, 1972; Eriksen \& Rohrbaugh, 1970) have favored a focusing type of selective process inasmuch as performance has been found to be a function not only of the total number of elements in the display but also of their physical proximity to each other.

In the present experiment, the number of noise elements in displays was kept constant at four and the spacing between the target letter and the noise elements was varied. The target letter was always located in one of the 12 clock positions and was flanked by two noise elements on each side. For the closest spacing (S-1), the target and noise elements occurred in consecutive clock positions on the circular display. For the second spacing (S-2), the target was separated by a blank clock position on each side before the noise elements began in consecutive clock positions. For S-3 spacing, a similar procedure was employed except there were two blank or vacant clock positions on each side of the target. In addition to the spacing variable, two kinds of noise elements were used. They were either an assortment of the four possible letters or black disks having a size approximately the same as that of the letters.

Unlike previous experiments where the position indicator appeared before the display, in the present experiment, the target letter and position indicator occurred simultaneously, followed by the noise elements at stimulus onset asynchronies (SOAs) that varied from 0 to $300 \mathrm{msec}$. This experimental manipulation was employed in an attempt to determine whether a focusing process distributed over time was involved in selective encoding.

If a gradual focusing was involved, it should begin with the appearance of the target letter and indicator. At zero or short SOAs, noise elements at the S-3 spacing would be expected to interfere as had been found in previous experiments. At somewhat longer SOAs, the focus would be more precise than to include noise elements at this spacing, and, therefore, their effect should cease. Noise elements at the S-2 spacing should continue to show interference at longer SOAs and noise elemerits at the S-1 spacing should be the last to approach 
LETTERS

s1

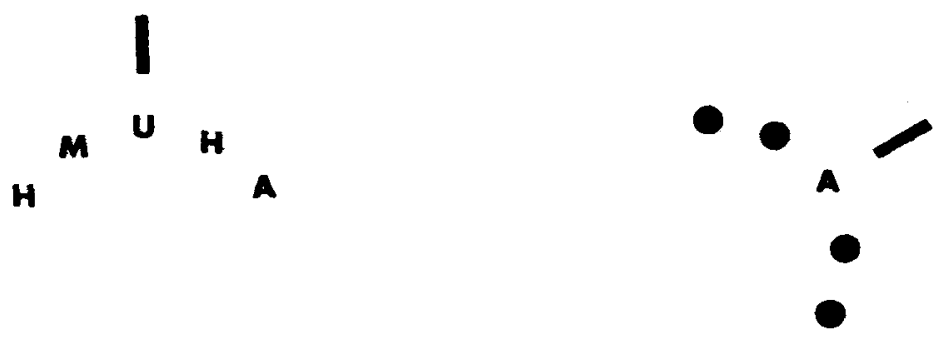

52

A $H$

$\mathbf{u}$

H

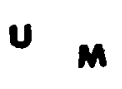

.

-

93

u

H

A

A

M

M

Fig. 1. Examples of the simulus displays with letters and dots as noise elements at each of the three levels of spacing.

asymptote on a RT-SOA function.

The use of two kinds of noise elements, letters or black disks, was a manipulation designed to provide information on how irrelevant elements in a display interfered with selective encoding.

\section{METHODS \\ Subject}

Four students, one male, served as paid Ss. One had served in a previous reaction-time experiment. All had normal or corrected to normal vision.

\section{Apparatus and Stimuli}

The stimuli were displayed with a Scientific- Prototype Model G-A three-field tachistoscope that had been modified by replacing the manufacturer's bulbs with Sylvania F4T5/CWX fluorescent lamps. All fields were set at $6 \mathrm{~mL}$. A black fixation cross $.6 \mathrm{deg}$ of visual angle mounted on a white vinyl card appeared in the adaptation field, which remained on except when Field II was activated. Trials were initiated by $S$ closing a switch that triggered the tachistoscope and a Hunter Model 120A KlocKounter. A microphone located below S's viewer triggered a Scientific Prototype Model 761-G audio threshold detection relay which stopped the KlocKounter. Voice reaction time was recorded in milliseconds.

The capital letters $A, H, U$, and $M$ were employed as stimuli. They were obtained from Paratipe No. 11316, and each letter subtended .2 deg of visual angle. These black letters were mounted on white vinyl cards. Target-letter cards were constructed by placing one of the four letters in a position corresponding to 1 of the 12 hour positions on an imaginary clock face having a diameter of $2 \mathrm{deg}$ of visual angle. Since each of the four letters occurred once in each of the 12 possible positions, a total of 48 target cards were involved. The cards containing the noise elements were constructed in the following way. For the case where the noise elements were a mixture of the four possible letters, a random assortment of these four letters were mounted on a plastic sheet in positions corresponding to the hour locations on a clock. For the S-1 spacing, the noise elements occurred in adjacent clock positions on each side of the target letter. In S-2, the nearest noise elements were a clock position removed from the target letter on each side, while in S-3 the nearest elements were two clock positions removed. In all cases, the noise elements were in adjacent clock positions to each other and two were present on each side of the target letter. Display examples are shown in Fig. 1 for each of the three spacings. These noise cards, when presented simultaneously with Field 1, gave the impression of the integrated five-letter display as shown in the examples in Fig. 1.

Where black disks were used as noise elements, the displays were constructed in a similar manner, except instead of letters, the noise elements were black disks subtending $.22 \mathrm{deg}$ of angle in diam. Their positions relative to the target letter were varied also over the three spacings. For both the letter and the disk condition, the distance of the nearest noise element to the target letter in degrees of angle was as follows: $s-1, .53 ; s-2,1.0$; and $s-3$, 1.4 .

The indicator was a black bar $.5 \mathrm{deg}$ of angle long and $.1 \mathrm{deg}$ of angle in width. It was placed on the same card as the target letter so that it occurred simultaneously with target appearance. It was positioned so that it fell outside the imaginary circle along which all display elements were arranged, and its nearest position was .5 deg of angle from the indicated letter. Further, it was positioned so that it would constitute an extension of an imaginary radius from the center of the display through the indicated position.

\section{Procedure}

Each $S$ served in two practice sessions followed by 12 experimental sessions. Following $5 \mathrm{~min}$ of dark adaptation, $\mathbf{S}$ was given eight practice trials on the first condition to be run that day. He was instructed to initiate a trial when a fixation cross appeared in good focus. At initiation, the adaptation field remained on with the onset of Field I, which contained the target letter and indicator. At SOAs of $0,50,100,150,200$, and $300 \mathrm{msec}$ after onset of Field $I$, the adaptation field terminated and was replaced by Fieid II, which contained the noise elements which, together with the 
target indicator in Field I, completed a five-element display. Fields I and II remained on together for 2 ec, allowing $S$ ample time to vocalize the indicated letter.

Alternate seasions were devoted to disks or letters as noise elements. Each session conmisted of six blocks of 24 trials each, one block under each of the six 8OAs. Within a block, the three spacing were represented equally often in a random order. Each letter occurred at each clock position under each of the three spacings equally often over blocks. The order of SOAs within a seasion was counterbalanced across restions for each type of noise. The designation of the initial experimental session as disks or letters was counterbalanced across Ss. Error rate averaged over Ss was approximately $2 \%$. Within this low error rate there was no systematic trend for errors to be greater under certain experimental conditions than others. Error trials were rerun at a later time in the experimental sessions so that each data point for each $S$ represents an average of 48 latencies.

\section{RESULTS}

Average RTs as a function of SOA, spacing between target and noise elements, and kind of noise elements (dots or letters) are shown in Fig. 2. A four-way analysis of variance (SOA, spacing, noive, and $\mathrm{Ss}$ ) was performed on these data. All main effects and interactions were significant at or beyond the .01 level except for the following: Spacing by Ss $[F(6,30)=$ $1.35, p>.25]$ and Spacing by Noise by Ss $[F(6,30)=1.8, p>.10]$. Separate analyses were performed on the data from the letter and dot conditions. For the letter condition, the only source failing to reach significance was the Spacing by Ss interaction. For the dot condition, the following failed to reach significance: spacing $[F(2,6)=1.38, p>.25]$, SOA by Spacing $[F(10,30)=1: 42$, $p>.10]$, and Spacing by Ss $[F(6,30)$ $=1.83, \mathrm{p}>.10$ ].

Inspection of Fig. 2 reveals that the significant effect of spacing within the letter noise condition is primarily due to S-1, which is the closest spacing. There is essentially no difference between the S-2 and S-3 spacing. The difference between $S-1$ and the other two spacings decreases as SOA increases, accounting for the significant SOA by Spacing interaction. For all spacings, RT is longest at simultaneous presentation of target and noise, as would be expected from previous work. Reaction time decreases monotonically with increasing SOA, approsching an asymptote between 150 and 200 msec.

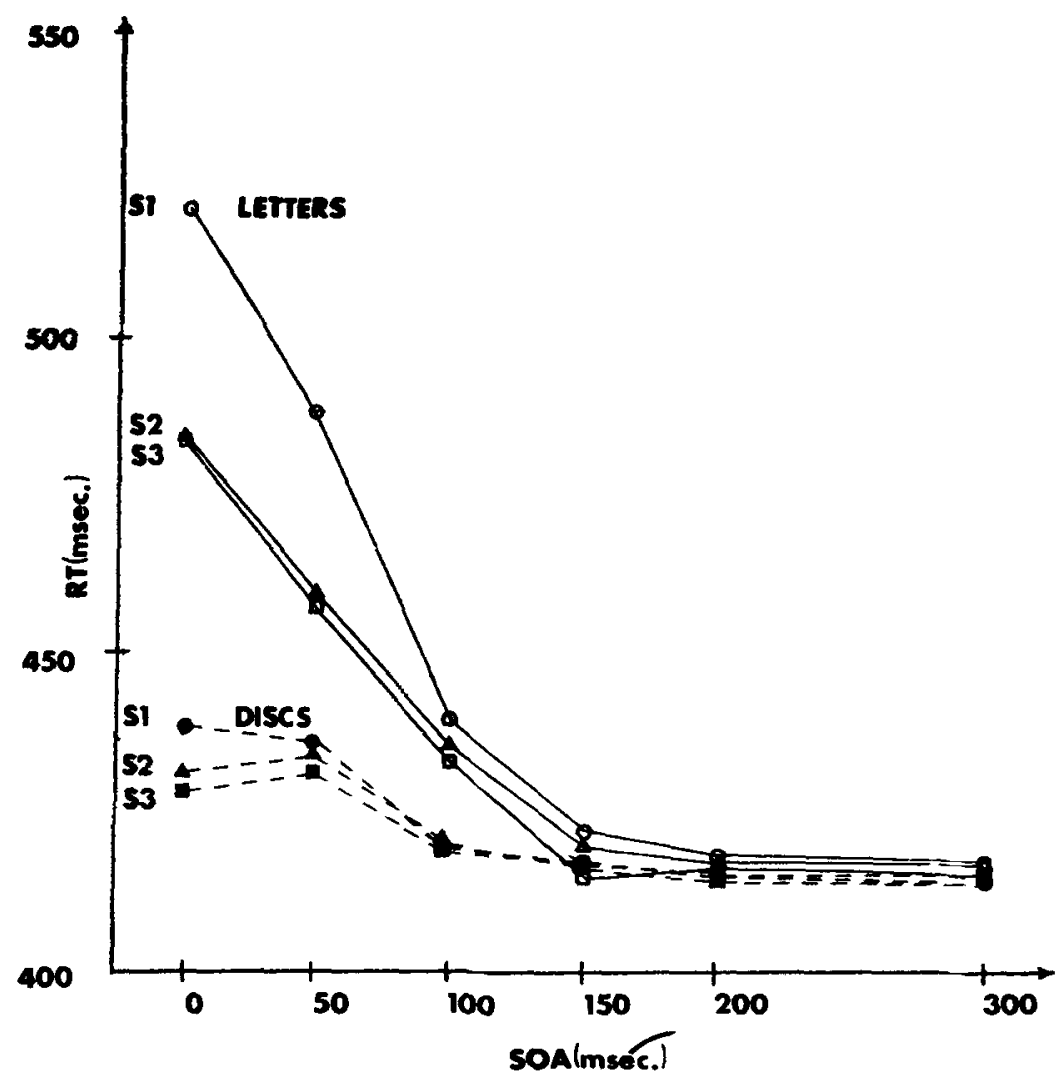

Fig. 2. Mean time to vocalize the target letter as a function of the SOA between the onset of the target and the onset of the noise elements. The parameters in the figure are for the two types of noise and their separation from the target letter.

The dots obviously provide less interference than the letters at all SOAs up until about 150 msec. The small but consistent effect of spacing was not significant, nor were any of the interactions involving spacing. Further inspection of Fig. 2 reveals that there is little drop in reaction time over the first $50 \mathrm{msec}$. The functions then appear to asymptote at $100 \mathrm{msec}$, and beyond $150 \mathrm{msec}$ curves from all conditions form a common asymptote.

\section{DISCUSSION}

The major interest of this experiment concerns the possibility of a gradual focusing process that underlies the selective encoding of the target stimulus. Results obtained with the spacing variable do not seem to fit such a conception. The obtained spacing effect is attributed solely to the difference between $S-1$ and the other two spacings. Once the noise elements are separated by a degree of angle or more from the target letter, further separation seems to be immaterial.

Also, the RT-SOA functions for the different spacings are not consistent with what would be expected from a gradual focusing process. These functions show no tendency to become asymptotic at different SOA values. As was pointed out above, a gradual focusing process would require not only that noise interference at the greatest spacing (S-3) cease at a shorter SOA than for S-2 and for S-2 at a shorter SOA than for $\mathbf{S - 1}$, but also at short SOAs there should be a RT difference among all three spacings.

The finding that spacing has an effect only when the noise elements are less than a degree of angle removed from the target would suggest that a contour interference or inhibition effect may be operating, much as has been suggested in the interpretation of visual masking studies. As an interpretation of the present results, however, this seems implausible for several reasons. First, the effect of adjacent contours on acuity have typically been found only when the interfering contours are less than $1 / 3$ of a degree of visual angle removed from the acuity target (Flom, Weymouth, \& Kahneman, 1963; this explanation would not account for why the disks produce much less interference in reaction time when Eriksen \& Rohrbaugh, 1970). Second, 
they are employed as the noise elements than do letters. Eriksen and Rohrbaugh (1970) had found that black disks had similar interference effects to letters when they were close enough to presumably impair contour formation.

Results obtained for the spacing variable may have to do with the nature of the attentional field. While James's (1890) characterization of attention as having a focus, a margin, and a fringe was a description at the phenomenal level, it may be that visual attention has a corresponding locus in the visual field that actually can be measured in terms of the degrees of visual angle it subtends. The analogy of James's characterization of attention to the characteristics of the eye are quite apparent. The focus of attention would correspond to the fovea, where there is a high resolution of detail, with the margin and fringe corresponding to the graduations in acuity that occur as areas of the retina progressively removed from the fovea are involved.

While the visual field varies in the degree of resolution or detail that is provided, the attentional field might be conceived as varying in the level of information processing or extraction that occurs. In the focus of the attentional field, stimuli are processed to a high level with considerable extraction of detail. Subtended into the visual field, this area of high-level information extraction may be no more than a degree of visual angle. Surrounding this high-level processing area may be an area where only gross information is extracted.

Under normal conditions of viewing, stimuli that are attended are fixated by the eye so that the small area of detailed information processing would correspond with the fovea where maximum detail is provided. This small area of high resolution and high-level processing is surrounded by the visual field where objects are less distinctly represented and constitute a somewhat vague indefinite background both in terms of preciseness in visual representations as well as in the attentional field.

This central processing mechanism, which we are calling attention, does not necessarily have to be locked to the eye in terms of its operation. That is, the attentional focus can be centered on a part of the visual field that does not correspond to the fovea. But, under these circumstances, the lack of resolution and detail for objects represented on the peripheral retina would provide very definite limits upon the level of information processing or extraction that could occur without a corresponding change in the eye's fixation. The duration of attention to stimuli in the peripheral field would be quite short, since the available information would be rapidly exhausted.

In terms of the results of the present experiment, if we consider the focus of attention as subtending approximately a degree of visual angle, we can account for the effect of the spacing variable in the following way.

When noise elements are approximately $1 / 2 \mathrm{deg}$ removed from the target, they fall in the area of high information extraction. There they are processed along with the target to the point of recognition or identification. Noise elements outside this focal area, on the other hand, receive a more gross level of processing, and their spacing from the target letter, as long as it is beyond a degree of angle separation, has little effect. Noise elements falling within the detailed processing area can affect reaction time by several different means. A noise element can achieve recognition on some trials more rapidly than the target element itself and thus either engage the encoding process so that the target is somewhat delayed from encoding or, in the case of a parallel encoding process, result in response competition between the two letters that have been identified.

Reaction time can also be slowed by a different means. If we envisage a limited-energy model, the processing of several letters to the point of identification or close to identification in a parallel fashion would require more energy or the use of more feature analyzing units, thus leaving less available for processing of the target letter. This, in turn, may result in a longer time before the target letter is processed to the encoding stage.

Noise elements beyond a degree separation from the target also can be expected to affect reaction time. These noise elements, even though processed less extensively, still require processing energy that would be subtracted from the energy available for target processing. But even here, the amount of complexity or information provided by these noise elements appears to have an effect. As seen in the present results, disks as noise elements impeded reaction time appreciably less than did letters. Also, their interfering effect appeared to have ended by $100 \mathrm{msec}$ as opposed to $150 \mathrm{msec}$ or more for the letter noise elements. This would suggest that even in the periphery of the attentional field there is a differential amount of information extraction, depending upon the detail afforded by the stimuli represented there. The finding that disks have less of an impairment effect upon reaction time might suggest that disks not only share fewer processing units with the target letter, but that the units they do share in common are shared early in the processing of the letter. By the time the letter is in the later stages of processing $(100 \mathrm{msec}$ or later), the processing units involved are no longer shared with those involved in processing disks.

\section{REFERENCES}

BARBER, P. J., \& FOLKARD, S. Reaction time under stimulus uncertainty with response certainty. Journal of Experimental Psychology, 1972, 93, 138-142.

ERIKSEN, C. W., \& ERIKSEN, B. A. Visual perceptual processing rates and backward and forward masking. Journal of Experimental Psychology, 1971, 89, 306-313.

ERIKSEN, C. W., \& ERIKSEN, B. A. Visual backward masking as measured by voice reaction time. Perception \& Psychophysics, 1972, 12, 5-8.

ERIKSEN, C. W., \& HOFFMAN, J. E. Some characteristics of selective attention in visual perception determined by vocal/reaction time. Perception \& Psychophysics, 1972, 11, 169-171.

ERIKSEN, C. W \& ROHRBAUGH, J. W Some factors determining efficiency of selective attention. The American Journal of Psychology, 1970,83,330-342.

FLOM, M. C., WEYMOUTH, F. W., \& KAHNEMAN, D. Visual resolution and contour interaction. Journal of the Optical Society of America, 1963, 53, 1026-1032

JAMES, W. The principles of psychology. I. New York: Dover, 1890.

RUMELHART, D. E. A multicomponent theory of the perception of briefly exposed visual displays. Journal of Mathematical Psychology, 1970, 7, $191-218$.

(Accepted for publication April 17, 1972.) 